\title{
A novel application of the white light/fringe projection duo: recovering high precision 3-D images from fossils for the digital preservation of morphology
}

\author{
Juan C. Gutiérrez-García, Tania A. Gutiérrez-García, J.F. Mosiño, \\ Ella Vázquez-Domínguez, Amalia Martínez, and Joaquín Arroyo-Cabrales
}

\begin{abstract}
There has been long historical interest in digital preservation of morphological features of biological materials, especially because often the preservation of sensitive molecules is critical for evolutionary studies. To this end, we successfully applied the recent advances of the fringe projection profilometry technique, in conjunction with white light and a new phase algorithm, to digitalize the shape of a fossil rodent hemimandible. We were able to generate a cloud of points in an array of data that allowed us to plot a three-dimensional (3-D) digital restoration of the entire fossil sample. The maximum resolution of this system is given by the diffraction limit (in the order of microns), and we show that this enhanced system can be used with objects in a range of 1-30 mm, minimizing the systematic errors induced by small vibrations or light fluctuations and, consequently, improving the signal-to-noise ratio of the recovered cloud data. This is a useful tool to preserve 3-D images of fossils and other biological objects for which rather detailed morphological information is required, like in research studies for biologists and paleontologists or, as in the present case, when researchers need a morphological replica because the sample will be destroyed for ancient DNA extraction.
\end{abstract}

Juan C. Gutiérrez-García. Centro de Investigaciones en Óptica, Loma del Bosque 115, A.P. 1-948, León, Guanajuato, 37150, México ing.juancarlosg@gmail.com

Tania A. Gutiérrez-García. Departamento de Ciencias Computacionales, Centro Universitario de Ciencias Exactas e Ingenierías, Universidad de Guadalajara, Blvd. Marcelino García Barragán 1421, Guadalajara, Jalisco, 44430, México taniagutierrezgarcia@gmail.com

J.F. Mosiño. Tecnológico Nacional de México - Instituto Tecnológico de León, Avenida Tecnológico S/N, León, Guanajuato, 37290, México jfmosino@gmail.com

Ella Vázquez-Domínguez. Departamento de Ecología de la Biodiversidad, Instituto de Ecología, Universidad Nacional Autónoma de México, Apartado Postal 70-275, Ciudad Universitaria, México Distrito Federal, 04510, México evazquez@ecologia.unam.mx Amalia Martínez. Centro de Investigaciones en Óptica, Loma del Bosque 115, A.P. 1-948, León, Guanajuato, 37150, México amalia@cio.mx Joaquín Arroyo-Cabrales. Laboratorio de Arqueozoología "M. en C. Ticul Alvarez Solórzano", Subdirección de Laboratorios y Apoyo Académico, Instituto Nacional de Antropología e Historia, Moneda \#16, Colonia Centro, 06060 Distrito Federal, México arromatu5@yahoo.com.mx

Gutiérrez-García, Juan C., Gutiérrez-García, Tania A., Mosiño, J.F., Vázquez-Domínguez, Ella, Martínez, Amalia, and ArroyoCabrales, Joaquín. 2015. A novel application of the white light/fringe projection duo: recovering high precision 3-D images from fossils for the digital preservation of morphology. Palaeontologia Electronica 18.2.6T: 1-13 palaeo-electronica.org/content/2015/1272-3d-fossil-images-and-white-light 
Keywords: morphometry; white light; rodent; 3-D imaging; fringe projection

\section{INTRODUCTION}

Different digital non-contact optical techniques have recently been developed, becoming popular for shape recovery (Geng, 2011) and fracture or deformation of three-dimensional (3-D) objects (Wang et al., 2010; Karpinsky and Zhang, 2012). One of these techniques used to recover shape is the fringe projection profilometry (FPP), in which a controlled fringe pattern of light is projected onto an object by using a digital video projector (DVP). The surface of an object causes deformation of the fringes, which are modulated into a phase domain; that is, by combining all the acquired frames we obtain an image with complex values, where the phase of each complex element is proportional to the corresponding pixel (px) height. Subsequently, the graphical representation (3-D surface topography) from this phase domain is obtained by different fringe pattern analysis methods, such as Fourier transform profilometry (FTP), phase shifting profilometry (PSP) or spatial phase detection (SPD) (Hu et al., 2006). PSP is the most effective and popular approach, widely used because of its robustness, simplicity and accuracy that enables the acquisition of complete field information at a very low cost, requiring few optical components to obtain detailed images only limited by the camera resolution ( $\mathrm{Hu}$ et al., 2006; Gorthi and Rastogi, 2010). PSP can be used with different types of electromagnetic sources (e.g., X-rays, IR-laser); however, when applied in conjunction with a low power electromagnetic source, like visible light (in this case, white light), it has an advantage that has been greatly underestimated: given that it is a contrast method instead of a radiative one, it is significantly harmless when used to obtain images of the surface of biological samples, as opposed to other sources such as X-ray and IR-light that irreversibly damage DNA (Goodhead, 1994; Weaver, 1995; Risom et al., 2003; Parplys et al., 2012) and induce mutations (Kielbassa et al., 1997; Agrawala et al., 2008). This property could be especially useful in cases where it is crucial to obtain high quality images from biological samples that include sensitive molecules, as is the case with ancient DNA (e.g., in genetic studies based on human fossil teeth from the Late Pleistocene; Chatters et al., 2014). The extraction of DNA from ancient samples necessarily requires the destruction of the sample (e.g., DNA is extracted from the tooth's root). In addition, because the availability of fossil samples is limited (fossilization is a rare phenomenon, thought to preserve only 1 to $5 \%$ of total biodiversity; Donovan and Paul, 1998) and fossils preserve key biological information, it is essential to conserve as many morphological features as possible (e.g., teeth and mandibles for rodents; Renaud et al., 2006, 2007). In this work, we successfully applied recent advances in the FPP technique to code the data of the shape of ancient rodent mandible samples into a digital format, samples that were later used for DNA extraction. We propose a simple new setup for fringe projection that adequately solves the PSP problems when combined with white light (e.g., miscalibrations, bias and detuning errors). We show that this enhanced technique can be used with very small objects, minimizing the systematic errors induced by small vibrations or light source fluctuations and, consequently, improving the signal-to-noise ratio of the recovered cloud data.

\section{MATERIALS AND METHODS}

\section{The Optical Arrangement}

The recovery of the surface characteristics of the sample was performed using the fringe projection profilometry method (principles are described in the Appendix). The optical system used (Figure 1) was composed of a laptop with a frame grabber attached to a multimedia LCD projector, with a converging lens of $10 \mathrm{~cm}$ focal length on a sliding base; on its right side we fitted a PixeLINK PLA741 CCD camera with a resolution of $1280 \times 1024$ pixels in grayscale ranging from 0 to 255 , with a f5/ 6 zoom lens and a rotary mounting.

The spatial resolution of the captured images was $53 \mathrm{px}$ per millimeter $(\mathrm{mm})$. The optical axis of the camera was perpendicular to the reference plane, while the line connecting the projector and camera were parallel to the reference plane accordingly to Huang et al. (2010). The distance between the CCD and the projector $d$ was $80 \mathrm{~mm}$, the distance between $C C D$ and the reference plane was $225 \mathrm{~mm}$, and between the projector and the reference plane was $239 \mathrm{~mm}$ (Figure 1). The entire system was set up in accordance with the sample dimensions (approximately $20 \mathrm{~mm}$ in 


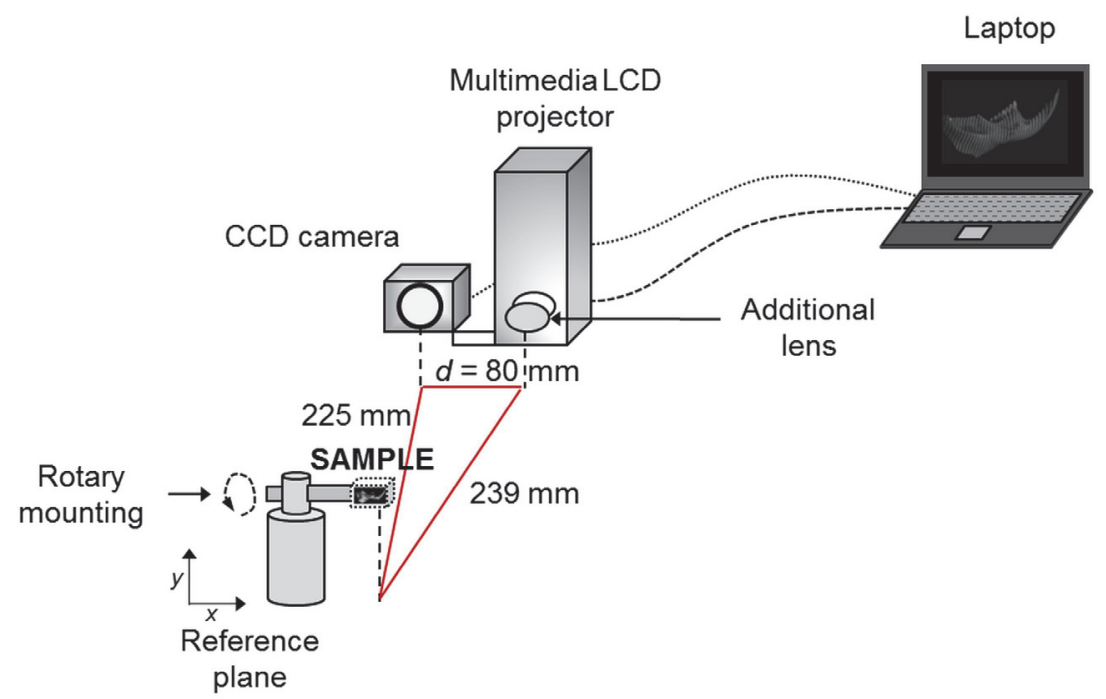

FIGURE 1. Optical set up specifications for fringe projection profilometry (FPP) used in this study for the recovery of a 3-D image of a hemimandible sample.

length, $15 \mathrm{~mm}$ in height and $3 \mathrm{~mm}$ in depth). All the calibrations of the optical setup were made following standard procedures (Zhang and Huang, 2006) and parameters, like gamma (Hoang et al., 2010), intensity (Yang and $\mathrm{He}, 2007$ ) and sinusoidal error (Gdeisat et al., 2009). The sample was fixed to the rotary platform by applying a delicate brushstroke with a paleontological consolidant (an adhesive routinely employed to repair or consolidate small fragments from crushed, broken or fragile samples), specifically used during the excavation of our fossil material (Arroyo-Cabrales and Alvarez, 1990); this substance worked as an interphase that joined the mounting with the farthest segment of the mandible. The consolidant was strong enough to firmly hold the hemimandible (weight $=0.16$ grams), and once dry, it was easily removed from the mounting and the sample. The sample was mounted on a rotating stage in order to enable $360^{\circ}$ scanning of the object for a reconstruction of a full 3-D image.

\section{Sample Preparation}

We used a semi-fossilized hemimandible from a rodent found inside the tropical cave Loltún, identified at that time to belong to the genus Ototylomys (Alvarez, 1982) (INAH number B6M13-19), which was used for DNA extraction after image acquisition (Gutiérrez-García et al., 2014). For the handling of the sample, we used protective clothing and face shields and took routine precautions to avoid human contamination of ancient samples (Hofreiter et al., 2001). Also, all instruments, equip- ment and the working area were sterilized with bleach, alcohol and/or ultraviolet (UV) radiation (Herrmann and Hummel, 1994). No other ancient or modern biological materials were ever used before in the working room.

\section{The Optical System and Procedure}

The non-linear nature of the FPP system introduces additional phase errors that can be compensated by using a large number of phase shift fringe patterns. However, this increases computational time and space, reducing the speed of the shape and accuracy of measurement. In addition, it depends directly on the available algorithms for calculating the wrapped phase (Yang and $\mathrm{He}$, 2007; Pan et al., 2009). We used an effective phase shifting algorithm (PSA), called MBE (Mainly Bias Error), that was recently developed specifically to prevent and even compensate for these systematic errors by using only eight frames to obtain the wrapped phase (Gutierrez-García et al., 2013).

As mentioned, modern or ancient DNA should not be exposed to high radiative coherent light sources because of their ionizing effect that is a damaging agent for DNA (Goodhead, 1994; Risom et al., 2003; Agrawala et al., 2008; Parplys et al., 2012). In order to minimize the exposure of the sample to white light (incoherent light), each of the eight frames needed for the MBE filter had an acquisition time of 20 milliseconds (ms), giving a short exposure time ( $<1$ second) for the complete optical system. Accordingly, we took eight frames 


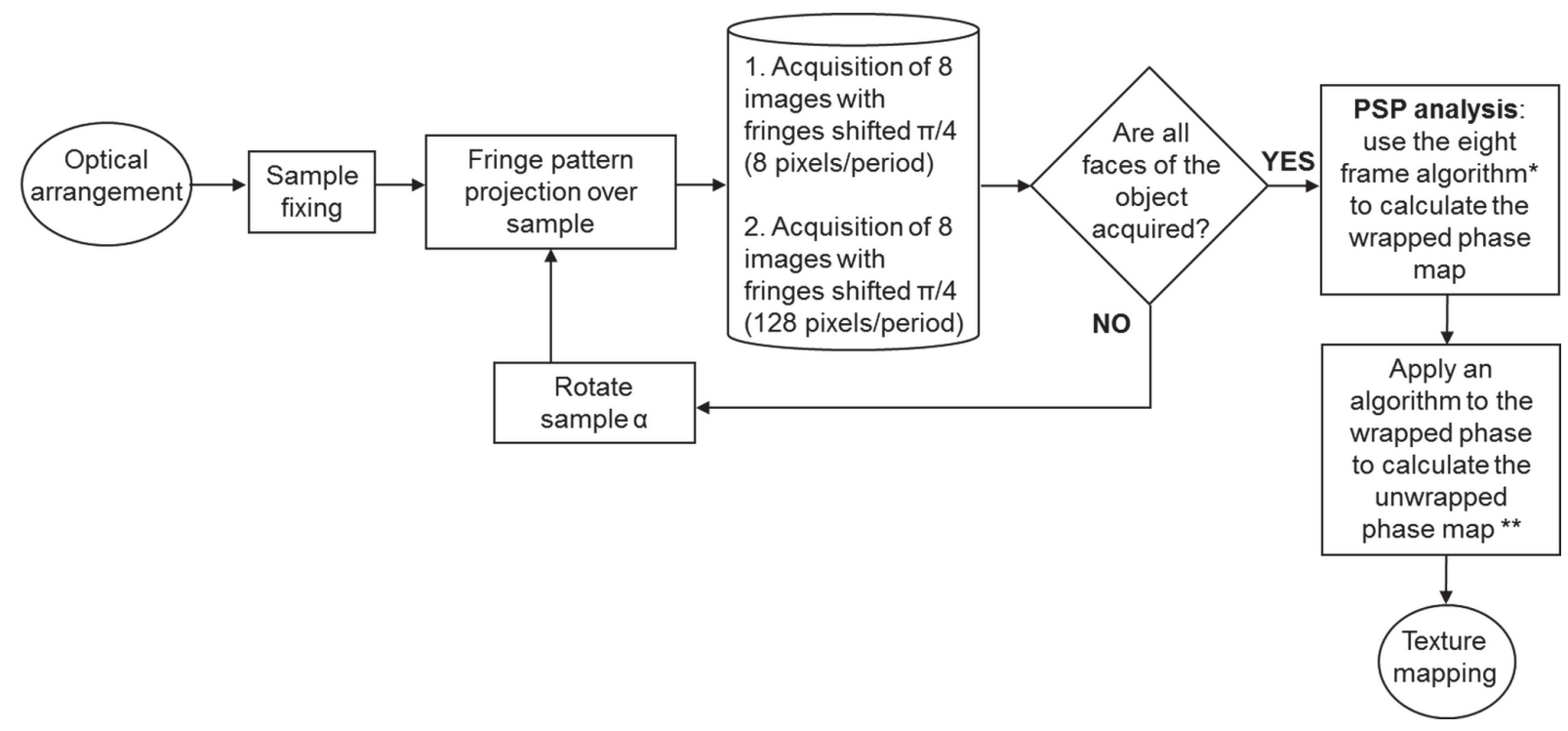

FIGURE 2. Flowchart of the 'OTY' procedure employed in this study, where: $\alpha=$ angle $\left(60^{\circ}\right.$ in this case), ${ }^{*}=\mathrm{MBE}$ algorithm (Gutiérrez-García et al., 2013), and ${ }^{* *}=$ Goldstein algorithm. OTY: name given to the white light system together with the phase shifting algorithm filter, based on the fact that it was developed for use on Ototylomys samples (see main text).

(spaced spectrally $\pi / 4$ ) with periods of 8 and 128 pixels per fringe $(0.125 \mathrm{~mm}$ and $2 \mathrm{~mm}$, respectively); subsequently, we rotated the sample $60^{\circ}$ on its own $x$-axis and acquired eight frames, repeating this until a full $360^{\circ}$ rotation had been completed. The use of both 8 and 128 px per fringe guarantees a successful detailed recovery of the sample's topography, overcoming the shadows resulting from the sample's irregular surface (Sansoni et al., 1999).

\section{Analysis}

Programs for image processing were implemented in MATLAB v7.12 and LabVIEW v8.2.1. After the gathering process and FPP technique to recover the wrapped phase image, we unwrapped the phase obtained with MBE by using Goldstein's branch cut algorithm, an extraordinarily fast method that requires little memory, successfully unwrapping the phase by minimizing its discontinuities (Goldstein et al., 1988; Ghiglia and Pritt, 1998). Once unwrapped, we estimated and subtracted the temporal carrier ( $\mathrm{Li}$ et al., 1998) to obtain the sample's reconstructed topography. Finally, we performed an image render and texturing of the 3-D image of the sample using MeshLab 1.3.3 (Cignoni et al., 2008). The image acquisition setup and the processing of data is shown in Figure 2. We named the white light system together with the phase shifting algorithm filter as "OTY", which are letters of Ototylomys, given that it was developed for use on samples of this genus.

\section{RESULTS}

We obtained a total of 96 frames from the Ototylomys hemimandible, divided in two sets of 48 frames that had 8 and $128 \mathrm{px} /$ period, respectively (magnitude maps obtained are shown in Figure 3 ).

In most optical methods, it is suggested to use a treatment of the sample (e.g., covering it with color) to ensure optimum performance and avoid reflections or other undesirable effects (Kaiser and Katterwe, 2001), like scattering, diffraction or dispersion, among others. However, a relevant advantage of our technique is that no such special sample preparation was required to obtain the frame or the processed images, a vital aspect to ensure the integrity of the sample (e.g., less risk of contamination by chemical agents or modern materials). The sequence followed to acquire the topographic maps included the fringes projection (Figure 4.1), the wrapped phase (Figure 4.2), the unwrapped phase (Figure 4.3) and the carrier compensation procedure (Figure 4.4). Image processing was performed without any spatial filtering to preserve the most detail in the final 3-D images (Figure 4.5).

The average resolution of the acquired images was $18.8 \mu \mathrm{m} / \mathrm{px}$. It should be noted that 


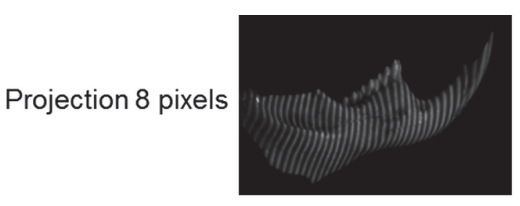

8 pixels

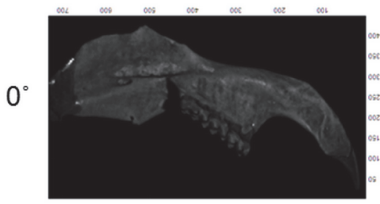

$60^{\circ}$

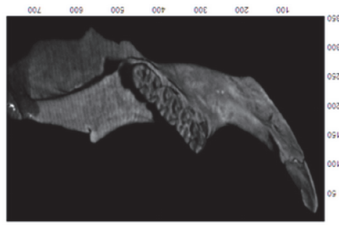

$120^{\circ}$

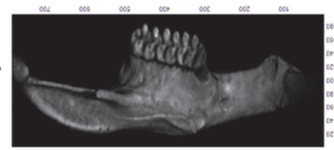

128 pixels
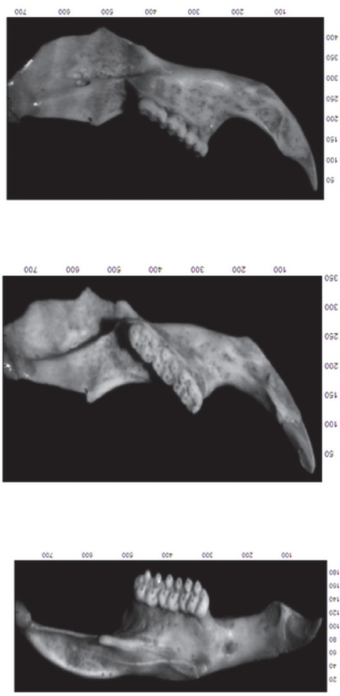

Projection 128 pixels

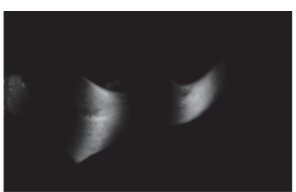

128 pixels

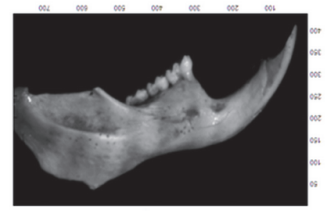

$240^{\circ}$
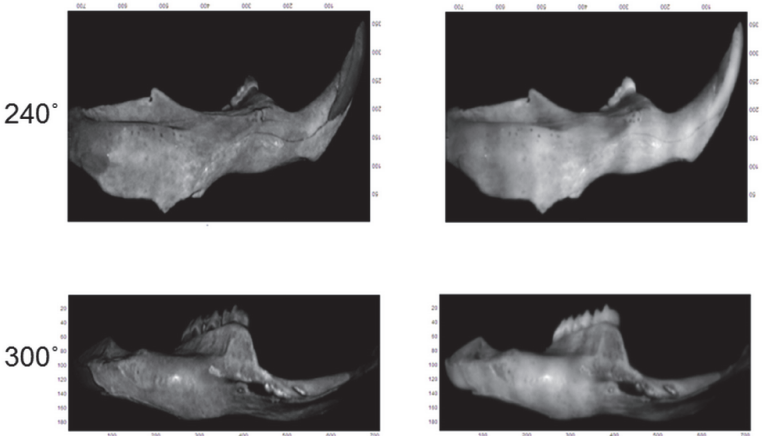

FIGURE 3. Magnitude maps of the sample obtained every $60^{\circ}$ for 8 and 128 pixels/period. The images show the resolution and detail levels given the number of fringes projected over the sample. The measurements' accuracy of surface and depth depends on the number of projected fringes, which include as many as the system can display (8 pixels/period for each fringe in this case). When the acquisition of details is difficult, a wider fringe is required (based on our sample size, we used 128 pixels).

this technique does not require the use of fiduciary markers for aligning different rotations of the object to attain the 3-D object, and we captured views every $60^{\circ}$ in order to scan the entire piece. Each of the six views processed with the optical system are shown in Figure 5, where it can be appreciated that some regions of the sample have no information. These regions are caused by the shadow created on the sample when projecting the fringes, reflecting the areas where no information can be obtained from the surface. However, the redundancy of the information is completed from all the set of images, which guarantees that each region is captured by the camera more than once; therefore, by combining each of the six views in a complete 3-D image, these regions are integrated and the missing gaps are filled (Figure 6.1). In addition, by using the topographic maps gathered from each of the six faces (at every $60^{\circ}$ angle) of the tested sample, we were able to construct the 3-D image including high fidelity dimensions and recovering all of its morphological details (Figure 6.2).

\section{DISCUSSION}

Several methods have been used to nondestructively image fossils in 3-D (Tafforeau et al., 2006; Sutton, 2008; Abel et al., 2012; Sánchez et al., 2013); however, most are based on ionizing radiation (e.g., X-rays, micro CT), which can result in molecular damage. A good comparison of these techniques was made by Cunningham et al. (2014), Rahman and Smith (2014) and Sutton et al. (2014), who discussed a wide variety of 3-D imaging techniques applicable to fossils and their suitability for different materials. When the crucial aim is to recover DNA from fossils, it is vital to use techniques that will cause as less molecular damage as possible, and those based on white light are a practical choice. Indeed, a segment of the white light spectrum has been used to describe the fossil's molecular content (Thomas, 2011).

Despite its advantages, the FPP system using white light has rarely been used to obtain 3-D images of biological samples. This method has only been explored via simulations under ideal laboratory conditions, mainly because of the complex- 
1)

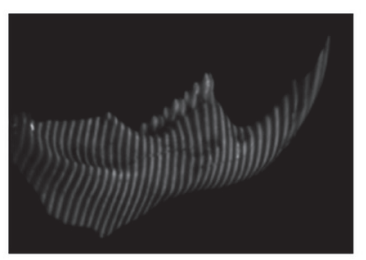

4)

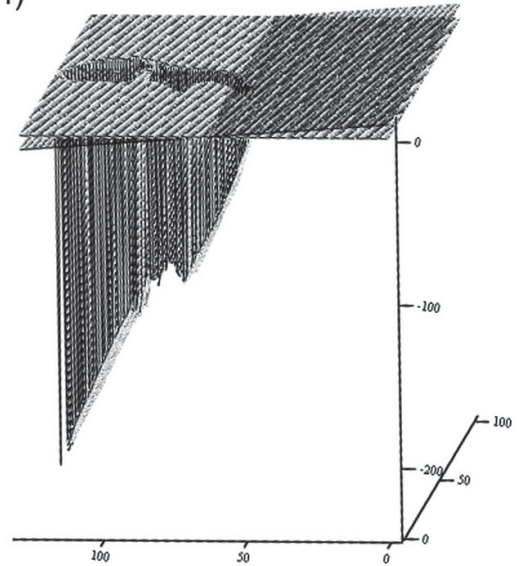

2)

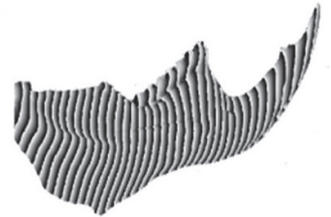

3)

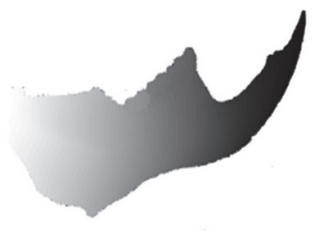

5)

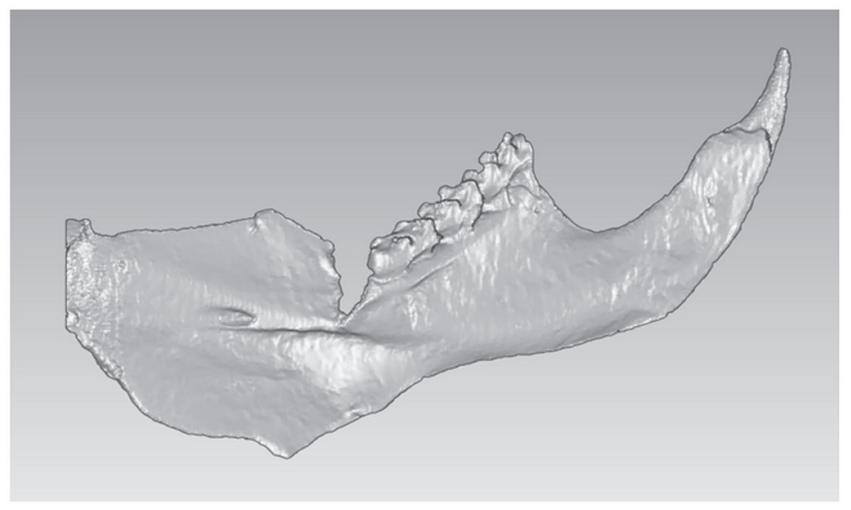

FIGURE 4. Process applied to recover the topography of the fossil sample. Where: 1) image captured by the CCD of the fringe projection on the sample, 2) wrapped phase obtained of the 8 frames after applying the MBE filter, 3) unwrapped phase map, 4) phase carrier compensation, and 5) surface of one of the views of the fossil recovered.

ities involved when working with it, including the need to consider specific features of the surface being analyzed, the optical setup requirements -like in the present case where, due to the fragility of the fossil, an optical rotation mount had to be designed to hold the piece-, potential bias errors, image acquisition defects and additional errors during image processing (Ghiglia and Pritt, 1998 and references therein; Kaiser and Katterwe, 2001).

In this study, we ensured the optimum performance of the system by adapting the optical setup to the sample size and by using the algorithm MBE (Gutiérrez-García et al., 2013), which reduces common errors during the processing of the frames, such as miscalibrations, bias and detuning errors. Because MBE is a real high bandwidth phase filter, it has the advantage that it minimizes the image's distortion and does not perform, like other common algorithms (e.g., Carré or $\mathrm{N}+1$ Bucket; by Carré and Hamon, 1966 and Surrel, 1993, respectively), as a low-pass filter. This final outcome yields the most accurate reconstruction of the object and with greater and enhanced detail than any other PSA that could be used for samples like our fossils. Moreover, the high 3-D image reso- lution we obtained $(18.8 \mu \mathrm{m})$ is comparable with those achieved with white light and well-known algorithms (e.g., $19 \mu \mathrm{m}$, Martínez et al., 2012). Furthermore, using this technique, the resolution is comparable with more expensive $3-D$ imaging techniques also used for fossils, as laser scanning $(>50 \mu \mathrm{m})$, magnetic resonance imaging $(>10 \mu \mathrm{m})$, neutron tomography $(>30 \mu \mathrm{m})$. Also, using a distinct lens it can achieve micro-CT resolution (>1 $\mu \mathrm{m})$ (Rahman and Smith, 2014; Sutton et al., 2014), considering that the current resolution limit for a white light image system is around $0.5 \mu \mathrm{m}$ because diffraction effects limit the maximum possible resolution (Conroy and Armstrong, 2005).

The camera exposure time is crucial to guarantee a high-intensity modulation of the captured fringe patterns along dark regions of the image, regardless of image saturation occurring on bright regions ( $\mathrm{Li}$ and Kofman, 2014). In our case, an exposure of $20 \mathrm{~ms}$ per frame was sufficient to avoid poor measurement accuracy and was also a short enough exposure to avoid harming the fossil, given that prolonged exposure to visible light can damage DNA (Goodhead, 1994; Kielbassa et al., 1997; Agrawala et al., 2008; Solarczyk et al., 2012). The different techniques and sequential 

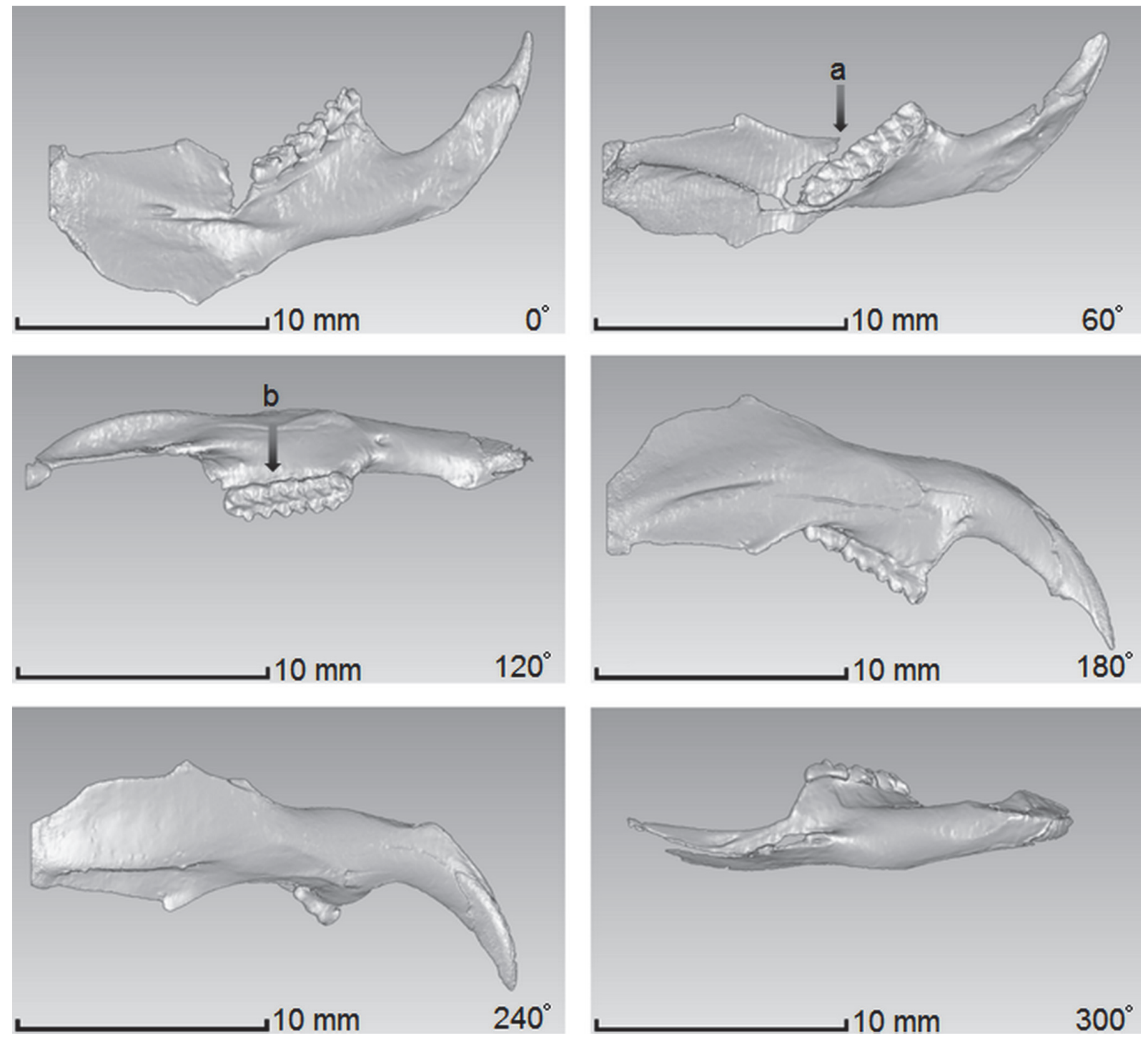

FIGURE 5. Processed views of a fossil sample acquired by the optical FPP system OTY. Each view is re-oriented $60^{\circ}$ degrees with respect to the prior. Where: "a" indicates a zone that has no information at $60^{\circ}$ in this stage of the process and will be corrected with the information of the next view, as is shown in "b".

steps we followed proved useful to obtain detailed measurements such as area, volume and distance between different points of the hemimandible, avoiding the need to manually handle the sample and the risk of damaging or contaminating it. Furthermore, the digital 3-D topography can be used in morphometric studies (e.g., Muñoz-Muñoz and Perpiñán, 2010), minimizing the errors associated with manual measurement tools. Moreover, it is useful for 2-D and 3-D studies such as images properties and color manipulation, geometric operations, segmentation analysis and linear and nonlinear filters for spatial and frequency-domain processing (Padmapriya and Vigneshnarthi, 2012), as shown in Figure 7.
Additional applications of our technique include basic morphological operations in 3-D images (distance transform, dilation, erosion, opening), 3-D restoration, cropping, padding, resizing, out-of-core image processing and active contour segmentation, just to mention a few. With this technique, scaled 3-D models and high-resolution images can be obtained for any fossil or sample, allowing their preservation in extreme detail, crucial in cases where they have to be destroyed. Due to the fact that this is a surface technique, there was no need to apply a high radiative energy source like laser scanning, or to produce a penetration depth like CT techniques. 


\section{GUTIÉRREZ-GARCÍA: 3D FOSSIL IMAGES AND WHITE LIGHT}
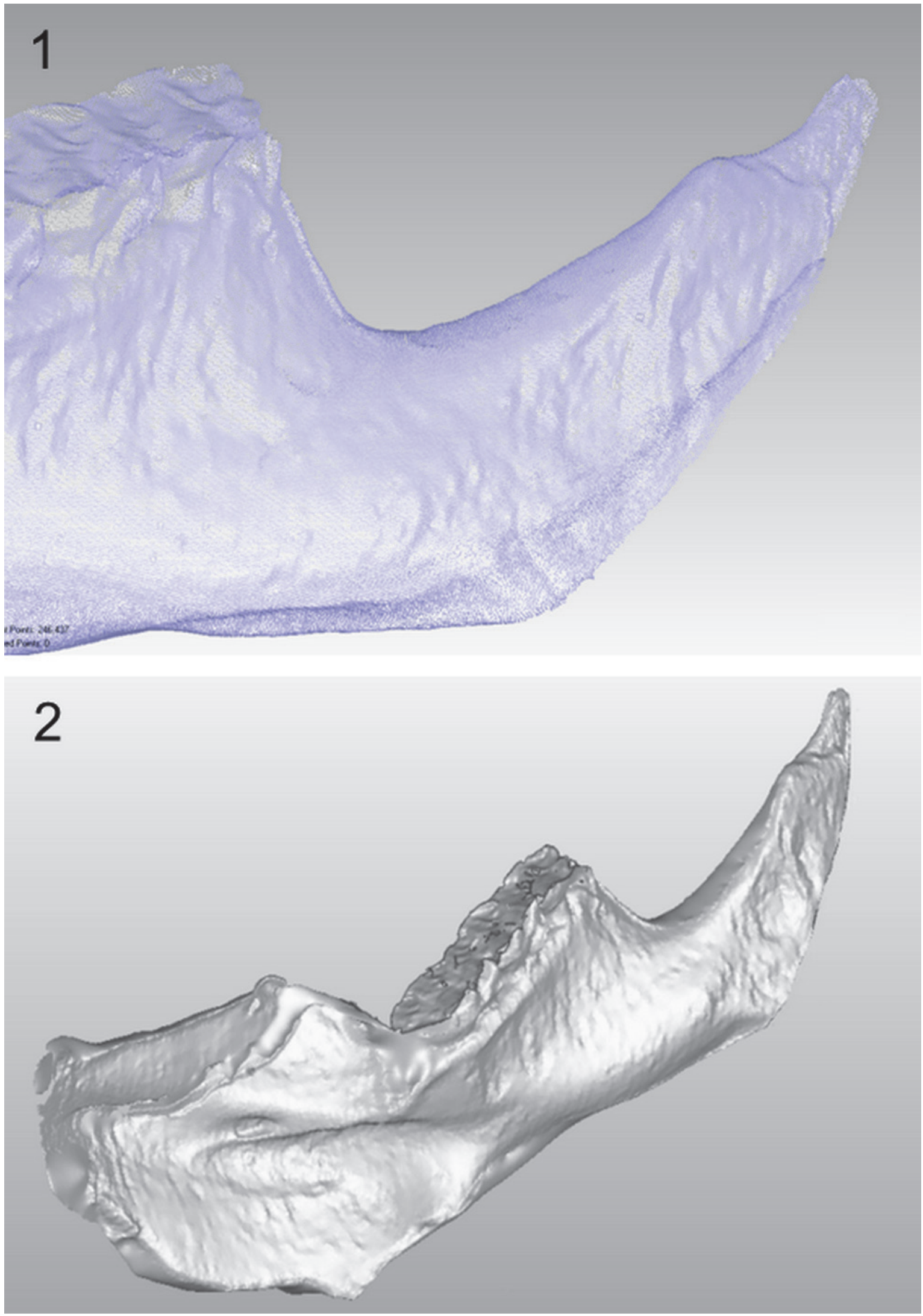

FIGURE 6. Full 3-D image of the reconstructed fossil after merging all the six views. 1) Cloud of points, 2) Final mesh. 

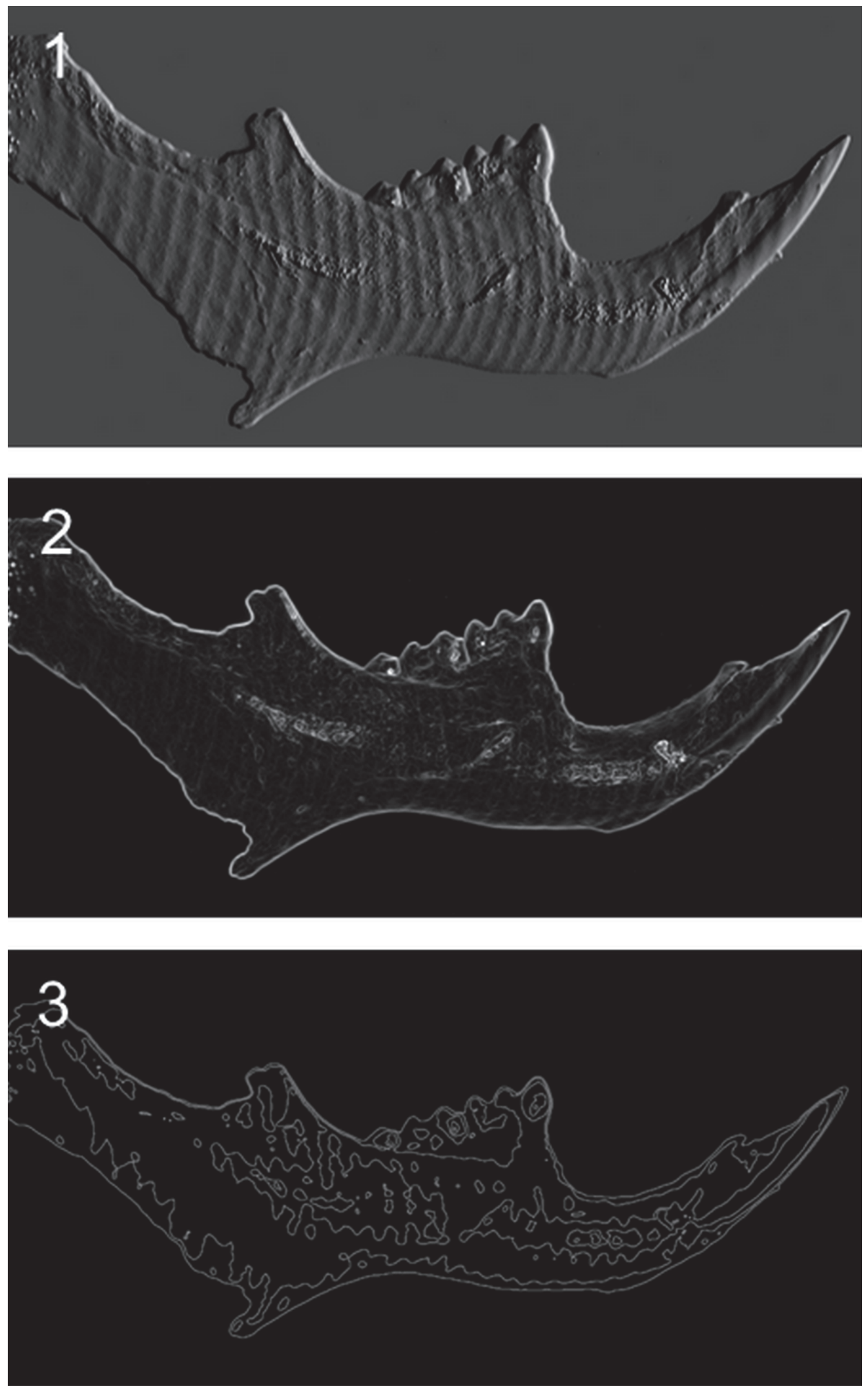

FIGURE 7. Examples of analyses that can be performed with the obtained data from the fossils: 1 ) denoting the relief (emboss filter), 2) detecting edges and transitions (sobel filter), 3) study of the roughness and waviness of a sample (topography filter). 
Furthermore, although the mean goal is to recover a higher resolution of a 3-D shape onto a cloud of data and to expose the sample the least possible to radiative energy, this method is rather simple and fast, can be performed in a small room and at a low cost since it does not require expensive equipment. The main advantages of this FPP technique in comparison with photogrammetry (PG) include: 1) it directly obtains the real topography's height distribution, while PG only acquires an estimation of it, 2) it obtains a complete cloud of points by using 48 frames, while PG needs 200 or more and only gets a dense cloud of points (e.g., Falkingham, 2012); the latter is because accuracy depends on the number of pictures taken, and 3) it obtains a 3-D exact image without any fiducially points, while for $P G$ the alignment of the required fiducial points directly impacts the exactitude of measuring and model scaling.

With our technique, we were able to fulfil the main aim of this work, to obtain 3-D images of fossils (and other biological objects), preserving at the same time key biological and detailed morphological information. It has a vast potential as application for ancient DNA and fossil studies where small to extremely small samples, as in our case, need to be destroyed for DNA extraction and molecular analysis. Finally, the images obtained with this technique can be used in a wide variety of studies, like comparing the anatomical features of extant and extinct organisms based on geometric morphometrics or to evaluate the tectonic deformation in fossils (Adams et al., 2004; Angielczyk and Sheets, 2007).

\section{ACKNOWLEDGEMENTS}

This research was partially funded by CONACyT (project 180449). J.C. Gutiérrez-García and T.A. Gutiérrez-García acknowledge CONACyT for the scholarship grants 207796 and 175434, respectively. The authors thank the Instituto Nacional de Antropología e Historia (INAH) for the loan of the fossil sample, and the Consejo de Arqueología, INAH for granting the permit to analyze the archaeological samples. We would like to dedicate this work to the memory of the archaeologist Ricardo Velázquez-Valadez, who was in charge of the major excavations in Loltún cave at the end of the $X X$ century and of the works for its preservation.

\section{REFERENCES}

Abel, R.L., Rettodini, C., and Richter, M. 2012. A palaeobiologist's guide to 'virtual' micro-CT preparation.
Palaeontologia Electronica, 15.2.6T:17pp; palaeoelectronica.org/content/issue-2-2012-technical-articles/233-micro-ct-workflow

Adams, D.C., Rohlf, F.J., and Slice, D.E. 2004. Geometric morphometrics: ten years of progress following the 'revolution'. Italian Journal of Zoology, 71:5-16.

Agrawala, P.K., Eschenbrenner, A., Penhoat, M.A.H.D., Boissiere, A., Politis, M.F., Touati, A., Sage, E., and Chetioui, A. 2008. Induction and repairability of DNA damage caused by ultrasoft X-rays: role of core events. International Journal of Radiation Biology, 84:1093-1103.

Alvarez, T. 1982. Restos de mamíferos recientes y pleistocénicos procedentes de las grutas de Loltún, Yucatán, México. Cuaderno de Trabajo 26. Instituto Nacional de Antropología e Historia, 26:7-35.

Angielczyk, K.D. and Sheets, H.D. 2007. Investigation of simulated tectonic deformation in fossils using geometric morphometrics. Paleobiology, 33:125-148.

Arroyo-Cabrales, J. and Alvarez, T. 1990. Restos óseos de murciélagos procedentes de las excavaciones en las grutas de Loltún. Instituto Nacional de Antropología e Historia, Colección Científica, 194:1-103.

Carré, P. and Hamon, J. 1966. Mesure interférentielle de la base géodésique du Bureau International des poids et mesures. Metrología, 2:143-150.

Chatters, J.C., Kennett, D.J., Asmerom, Y., Kemp, B.M., Polyak, V., Blank, A.N., Beddows, P.A., Reinhardt, E., Arroyo-Cabrales, J., Bolnick, D.A., Malhi, R.S., Culleton, B.J., Erreguerena, P.L., Rissolo, D., Morell-Hart, S., and Stafford, T.W. 2014. Late Pleistocene human skeleton and mtDNA link paleoamericans and modern native americans. Science, 344:750-754.

Cignoni, P., Corsini, M., and Ranzuglia, G. 2008. MeshLab: an open-soirce 3D mesh processing system. ERCIM News, 73:47-48.

Conroy, M. and Armstrong, J. 2005. A comparsion of surface metrology techniques. Journal of Physics, 13:458-465.

Cunningham, J.A., Rahman, I.A., Lautenschlager, S., Rayfield, E.J., and Donoghue, C.J. 2014. A virtual world of paleontology. Trends in Ecology \& Evolution, 29:347-357.

Donovan, S.K. and Paul, C.R. 1998. The Adequacy of the Fossil Record. John Wiley and Sons, England.

Falkingham, P.L. 2012. Acquisition of high resolution 3D models using free, open-source, photogrammetric software. Palaeontologia Electronica,15.1.1T:15pp; palaeo-electronica.org/content/issue-1-2012-technical-articles/92-3d-photogrammetry

Gdeisat, M.A., Abid, A., Burton, D.R., Lalor, M.J., Lilley, F., Moore, C., and Qudeisat, M. 2009. Spatial and temporal carrier fringe pattern demodulation using the one-dimensional continuous wavelet transform: recent progress, challenges, and suggested software. Optics and Lasers in Engineering, 47:13481361. 
Geng, J. 2011. Structured-light 3D surface imaging: a tutorial. Advances in Optics and Photonics, 3:128160.

Ghiglia, D.C. and Pritt, M.D. 1998. Two-Dimensional Phase Unwrapping: Theory, Algorithms, and Software (first edition). John Wiley and Sons, Ohio.

Goldstein, R.M., Zebker, H.A., and Werner, C.L. 1988. Satellite radar interferometry: two-dimensional phase unwrapping. Radio Science, 23:713-720.

Goodhead, D.T. 1994. Initial events in the cellular effects of ionizing radiations: clustered damage in DNA. International Journal of Radiation Biology, 65:7-17.

Gorthi, S.S. and Rastogi, P. 2010. Fringe projection techniques: whither we are? Optics and Lasers in Engineering, 48:133-140.

Gutiérrez-García, J.C., Mosiño, J.F., Martínez, A., Gutiérrez-García, T.A., Vázquez-Domínguez, E., and Arroyo-Cabrales, J. 2013. Practical eight-frame algorithms for fringe projection profilometry. Optics Express, 21:903-917.

Gutiérrez-García, T.A., Vázquez-Domínguez, E., ArroyoCabrales, J., Kuch, M., Enk, J., King, C., and Poinar, H.N. 2014. Ancient DNA and the Tropics, a rodent's tale. Biology Letters, 10:20140224.

Herrmann, B. and Hummel, S. 1994. Ancient DNA, Recovery and Analysis of Genetic Material from Paleontological, Archaeological, Museum, Medical and Forensic Specimens. Springer-Verlag, New York.

Hoang, T., Pan, B., Nguyen, D., and Wang, Z. 2010 Generic gamma correction for accuracy enhancement in fringe-projection profilometry. Optics Letters, 35:1992-1994.

Hofreiter, M., Serre, D., Poinar, H.N., Kuch, M., and Pääbo, S. 2001. Ancient DNA. Nature Reviews Genetics, 2:353-359.

Hu, Y., Xi, J., Chicharo, J., and Yang, Z. 2006. Improved three-step phase shifting profilometry using digital fringe pattern projection. Computer Graphics, Imaging and Visualization, 2006 International Conference on, IEEE:161-167.

Huang, L., Chua, P.S.K., and Asundi, A. 2010. Leastsquares calibration method for fringe projection profilometry considering camera lens distortion. Applied Optics, 49:1539-1548.

Kaiser, T.M. and Katterwe, H.K. 2001. The application of 3D-microprofilometry as a tool in the surface diagnosis of fossil and sub-fossil vertebrate hard tissue. An example from the Pliocene upper laetolil beds, Tanzania. International Journal of Osteoarchaeology, 11:350-356.

Karpinsky, N. and Zhang, S. 2012. High-resolution, realtime 3D imaging with fringe analysis. Journal of RealTime Image Processing, 7:55-66.

Kielbassa, C., Roza, L., and Epe, B. 1997. Wavelenght dependence of oxidative DNA damage induced by UV and visible light. Carcinogenesis, 18:811-816.

Li, J.L., Su, X.Y., Su, H.J., and Cha, S.S. 1998. Removal of carrier frequency in phase-shifting techniques. Optics and Lasers in Engineering, 30:107-115.
Li, D. and Kofman, J. 2014. Adaptive fringe-pattern projection for image saturation avoidance in 3D surfaceshape measurement. Optics Express, 22:9887-9901.

Ma, S., Quan, C., Zhu, R., and Tay, C.J. 2012. Investigation of phase error correction for digital sinusoidal phase-shifting fringe projection profilometry. Optics and Lasers in Engineering, 50:1107-1118.

Martínez, A., Rayas, J.A., Cordero, R.R., Balieiro, D., and Labbe, F. 2012. Leaf cuticle topography retrieved by using fringe projection. Optics and Lasers in Engineering, 50:231-235.

Muñoz-Muñoz, F. and Perpiñán, D. 2010. Measurement error in morphometric studies: comparison between manual and computerized methods. Annales Zoologici Fennici, 47:46-56.

Padmapriya, A. and Vigneshnarthi, S. 2012. Image processing operations for $3 \mathrm{~d}$ image. International Journal of Scientific and Research Publications, 2:1-6.

Pan, B., Kemao, Q., Huang, L., and Asundi, A. 2009. Phase error analysis and compensation for nonsinusoidal waveforms in phase-shifting digital fringe projection profilometry. Optics Letters, 34:416-418.

Parplys, A.C., Petermann, E., Petersen, C., Dikomey, E., and Borgmann, K. 2012. DNA damage by X-rays and their impact on replication processes. Radioterapy and Oncology, 102:466-471.

Rahman, I.A. and Smith, S.Y. 2014. Virtual Paleontology: computer aided analysis of fossil form and function. Journal of Paleontology, 88:633-635.

Rathjen, C. 1995. Statistical properties of phase-shift algorithms. Journal of the Optical Society of America A, 12:1997-2008.

Renaud, S., Auffray, J.C., and Michaux, J. 2006. Conserved phenotypic variation patterns, evolution along lines of least resistance, and departure due to selection in fossil rodents. Evolution, 60:1701-1717.

Renaud, S., Chevret, P., and Michaux, J. 2007. Morphological vs. molecular evolution: ecology and phylogeny both shape the mandible of rodents. Zoologica scripta, 36:525-535.

Risom, L., Moller, P., Vogel, U., Kristjansen, P.E., and Loft, S. 2003. X-ray-induced oxidative stress: DNA damage and gene expression of HO-1, ERCC1 and OGG1 in mouse lung. Free Radical Research, 37:957-966.

Sánchez, S., Fernandez, V., Pierce, S.E., and Tafforeau, P. 2013. Homogenization of simple absorption for the imaging of large and dense fossils with synchrotron microtomography. Nature Protocols, 8:1708-1717.

Sansoni, G., Carocci, M., and Rodella, R. 1999. Threedimensional vision based on a combination of graycode and phase-shift light projection: analysis and compensation of the systematic errors. Applied Optics, 38:6565-6573.

Solarczyk, K.J., Zarebski, M., and Dobrucki, J.W. 2012. Inducing local DNA damage by visible light to study chromatin repair. DNA Repair, 11:996-1002.

Surrel, Y. 1993. Phase stepping: a new self-calibrating algorithm. Applied Optics, 32:3598-3600. 
Sutton, M., Rahman, I., and Garwood, R. 2014. Techniques for Virtual Palaeontology. John Wiley and Sons, England.

Sutton, M.D. 2008. Tomographic techniques for the study of exceptionally preserved fossils. Proceedings of the Royal Society B, 275:1587-1593.

Tafforeau, P., Boistel, R., Boller, E., Bravin, A., Brunet, M., Chaimanee, Y., Cloetens, P., Feist, M., Hoszowska, J., Jaeger, J.-J., Kay, R.F., Lazzari, V., Marivaux, L., Nel, A., Nemoz, C., Thibault, X., VIgnaud, P., and Zabler, S. 2006. Applications of X-ray synchrotron microtomography for non-destructive 3D studies of paleontological specimens. Applied Physics A, 83:195-202.

Takeda, M. and Mutoh, K. 1983. Fourier transform profilometry for the automatic measurement of 3-D object shapes. Applied Optics, 22:3977-3982.
Thomas, D.B. 2011. Illuminating fossils by NIR. NIR News, 22:6-8.

Wang, Z., Nguyen, D.A., and Barnes, J.C. 2010. Some practical considerations in fringe projection profilometry. Optics and Lasers in Engineering, 48:218225.

Weaver, D.T. 1995. What to do at an end: DNA doublestrand-break repair. Trends in Genetics, 11:388-392.

Yang, F. and He, X. 2007. Two-step phase-shifting fringe projection profilometry: intensity derivative approach. Applied Optics, 46:7172-7178.

Zhang, S. and Huang, P.S. 2006. Novel method for structured light system calibration Optical Engineering, 45:083601-8. 


\section{APPENDIX}

\section{PRINCIPLE OF FRINGE PROJECTION}

In a fringe projection profilometry system (FPP), a series of straight, vertical or horizontal and equally spaced fringes are generated by computer and projected onto the surface of an object using a digital video projector. At the same time, a CCD (charged-coupled device) camera captures the intensity of the fringes from the surface of the object for later processing. By using the phase shifting (PS) method, "N" fringe patterns of frames that were captured by the camera, each nth intensity pattern $I_{n}(x, y)$ can be commonly described as:

$I_{n}(x, y)=a(x, y)+b(x, y) \cos [(2 \pi / p) x+\varphi(x, y)+\theta \cdot n] ; \mathrm{n}=1 \ldots \mathrm{N}$

where $N$ is the total number of images acquired; $n$ is the number of the phase-shifting steps, $(x, y)$ denotes the coordinates of an arbitrary point in the object being analyzed; $p$ is the period of the equally spaced fringes on the reference plane; $\varphi(x, y)$ is the phase map related to the object profile, also called wrapped phase; and $\theta$ is the assigned phase shift value that is usually equal to $2 \pi / N$ (Ma et al., 2012).

The numerical process to recover the wrapped phase from the set of captured images is performed with a phase shift algorithm (PSA). The correct design of this algorithm, in accordance with a specific setup, determines the complexity and resolution of the results that, briefly, uses the intensity values obtained by shifting the fringes on the object to calculate the phase (Rathjen, 1995). Next, an unwrapping procedure is needed to make this phase map continuous, which is done by removing the artificial discontinuities added by the FPP technique. This phase's unwrapping is a complex process and the techniques used to perform it assume that the neighboring pixel differences of the unwrapped phase can be estimated by adding an integral multiple of $2 \pi$, when these differences are less than $\pi$. Using some mathematical methods it is possible to obtain a cloud of data points that is proportional to the analyzed object (Ghiglia and Pritt, 1998). After this, the temporal carrier is calculated and subtracted (Li et al., 1998) and the topography or profile is calculated by:

$h(x, y)=I_{0} \cdot \varphi(x, y) /\left[2 \pi f_{0} d+\varphi(x, y)\right]$

where $I_{0}$ is the distance between the CCD camera and the reference plane, and $d$ is the distance between the camera and the projector (Takeda and Mutoh, 1983). 